\title{
Combined central retinal vein, central retinal artery and cilioretinal artery occlusion with ischemic macular hole secondary to severe orbital cellulitis after black fly bite
}

This article was published in the following Dove Press journal:

International Medical Case Reports Journal

\author{
Narumon Keorochana' \\ Sritatath Vongkulsiri ${ }^{2}$ \\ Raveewan Choontanom ${ }^{3}$ \\ 'Ocular Inflammatory and Uveitis \\ Division, Department of Ophthalmology, \\ Phramongkutklao Hospital, \\ Phramongkutklao College of Medicine, \\ Bangkok, Thailand; ${ }^{2}$ Retina Division, \\ Department of Ophthalmology, \\ Phramongkutklao Hospital, \\ Phramongkutklao College of Medicine, \\ Bangkok, Thailand; ${ }^{3}$ Oculoplastic and \\ Reconstruction Division, Department of \\ Ophthalmology, Phramongkutklao \\ Hospital, Phramongkutklao College of \\ Medicine, Bangkok, Thailand
}

Background: Orbital cellulitis is an infection of the ocular adnexal and orbital tissues behind the orbital septum. The complications are poor prognosis. This paper is to describe some severe rare complications of orbital cellulitis.

Case presentation: A case of 32-year-old female presented with acute severe progressive orbital cellulitis on the right eye after a black fly (Simuliidae spp.) bite. Orbital imaging with computed tomography and magnetic resonance imaging showed eyelid abscess with orbital abscess formation and superior ophthalmic vein thrombosis. She was promptly treated with intravenous vancomycin, piperacillin and had surgical procedures to release orbital pressure including lateral canthotomy, lateral cantholysis, and eyelid with orbital abscess drainage. Pus collection culture was positive for Staphylococcus aureus. Even though she responded well to antibiotics treatment, progression involved severe orbital inflammation with marked increased orbital pressure, and her visual acuity was such that she had no light perception. Combined central retinal vein, central retinal artery and cilioretinal artery occlusion were identified. Fundus examination showed retinal whitening at the posterior pole and a few scattered flame-shaped retinal hemorrhages. The cilioretinal artery presented with perivascular retinal whitening. A fundus fluorescein angiogram revealed cilioretinal and retinal artery filling delay and also delayed arteriovenous transit time. She also developed an ischemic macular hole after a week of treatment. We demonstrate an optical coherence tomography image showing the intact posterior hyaloid membrane that represents no traction on the macula to support the ischemic cause.

Conclusion: Multiple retinal vascular occlusion and ischemic macular hole could be an early complication of severe orbital cellulitis from increased orbital pressure; therefore, close monitoring of visual acuity with IOP, and prompt treatment when indicated might reduce the risk of consequent blindness.

Keywords: black fly, orbital cellulitis, central retinal vein occlusion, central retinal artery occlusion, cilioretinal artery occlusion, macular hole

\section{Background}

Orbital cellulitis is an infection of the ocular adnexal and orbital tissues behind the orbital septum including fat and ocular muscles. It is caused by the spreading of infection from the paranasal sinuses, eyelid skin or by hematogenous spread. The most common pathogens are Gram-positive bacteria such as Streptococcus spp. and Staphylococcus aureus. ${ }^{1,2}$ Fungal infections should be
Ocular Inflammatory and Uveitis Division, Department of Ophthalmology, Phramongkutklao Hospital, 3 I5

Ratchvidhi Rd., Thung Phayathai, Ratchathewi, Bangkok 10400, Thailand

Tel +6627639300

Fax +6623549303

Email narumon_keo@hotmail.com 
considered in immunocompromised patients. The complications of orbital cellulitis are poor prognosis. ${ }^{3}$

In this paper, we would like to describe a severe case of orbital cellulitis and abscess following an insect bite. In addition, many rare complications occurred in this case such as multiple retinal vascular occlusion, ischemic macular hole, superior ophthalmic vein thrombosis, and ocular myopathy. We investigate computed tomography (CT) scan and magnetic resonance imaging (MRI) with contrast of the brain and orbit to show the detail of complications. Also, we provide fundus fluorescein angiography (FFA) and optical coherence tomography (OCT) images to explain the mechanism of those diseases. We describe this case following the CARE guideline. ${ }^{4}$

\section{Case report}

A 32-year-old female from Phrae, a province in Northern Thailand, was bitten by black fly on the right upper eyelid. She reported minimal eye pain, itchy and irritated at right upper eyelid and vision was normal. She received oral cloxacillin and intravenous chlorpheniramine from a primary care hospital on the same day. On the next day, she went to a provincial hospital because her condition had worsened. She was admitted and diagnosed with preseptal cellulitis of the right eye. Intravenous cloxacillin was prescribed. She showed no improvement but progressive orbital swelling and severe pain. She was referred to a tertiary care hospital on the following day.

On her presentation at Phramongkutklao hospital, she had suffered from progressive proptosis and ocular pain for 2 days. The right eyelid appeared with marked swelling, was tense, with erythema and tenderness and was unable to be opened (Figure 1). The anterior segment, fundus, and IOP could not be evaluated, and nor could visual acuity. The left eye was unremarkable and had visual acuity and IOP of 20/20 and 15 respectively. High blood pressure, $142 / 97 \mathrm{mmHg}$, and body temperature of $36.5^{\circ} \mathrm{C}$ were noted. General physical examination was normal.

Computed tomography (CT) scan with contrast of the brain and orbit (Figure 2) revealed extensive right orbital inflammation with abscess formation, which extended medially from the extraconal into the intraconal space posterior to the eyeball.

Magnetic resonance imaging (MRI) with contrast of the brain and orbit (Figure 3) showed multiple small lesions of hyposignal intensity and rim-enhancement occupying at preseptal and intraconal regions. The right superior ophthalmic vein thrombosis was suspicious by the evidence in coronal MRI (Figure 3C and D) showing no intraluminal contrast filling with rim enhancement.

Intraoperative findings showed soft tissue necrosis around the bitten site with orbital abscess. Surgical procedures to release orbital pressure including lateral canthotomy, lateral cantholysis, wound exploration, debridement, and orbital abscess drainage were performed. Pus was sent for Gram staining, a $\mathrm{KOH}$ test, and culture for bacteria and fungus. The Gram stain was positive for Gram-positive cocci, and numerous polymorphonuclear cells. The bacterial culture was later reported to contain Staphylococcus

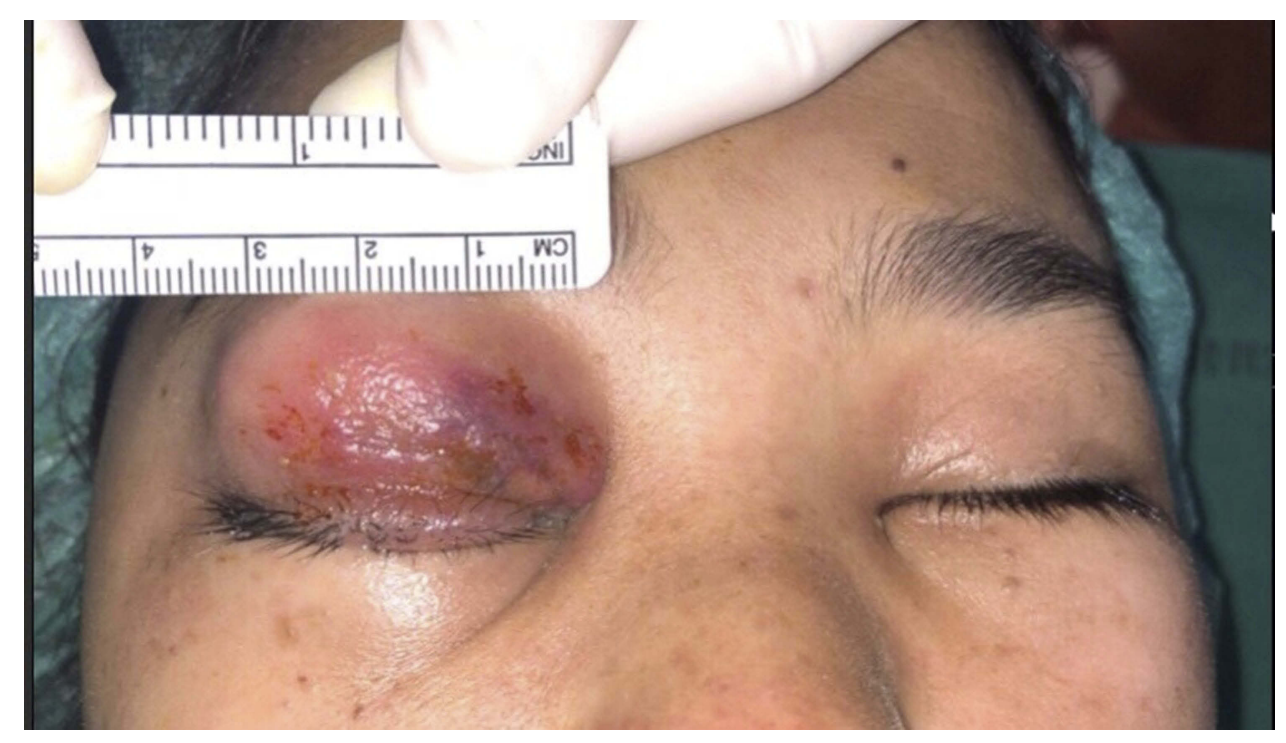

Figure I Day 3 marked swelling, erythematous tense right eyelid presented at the first presentation. 


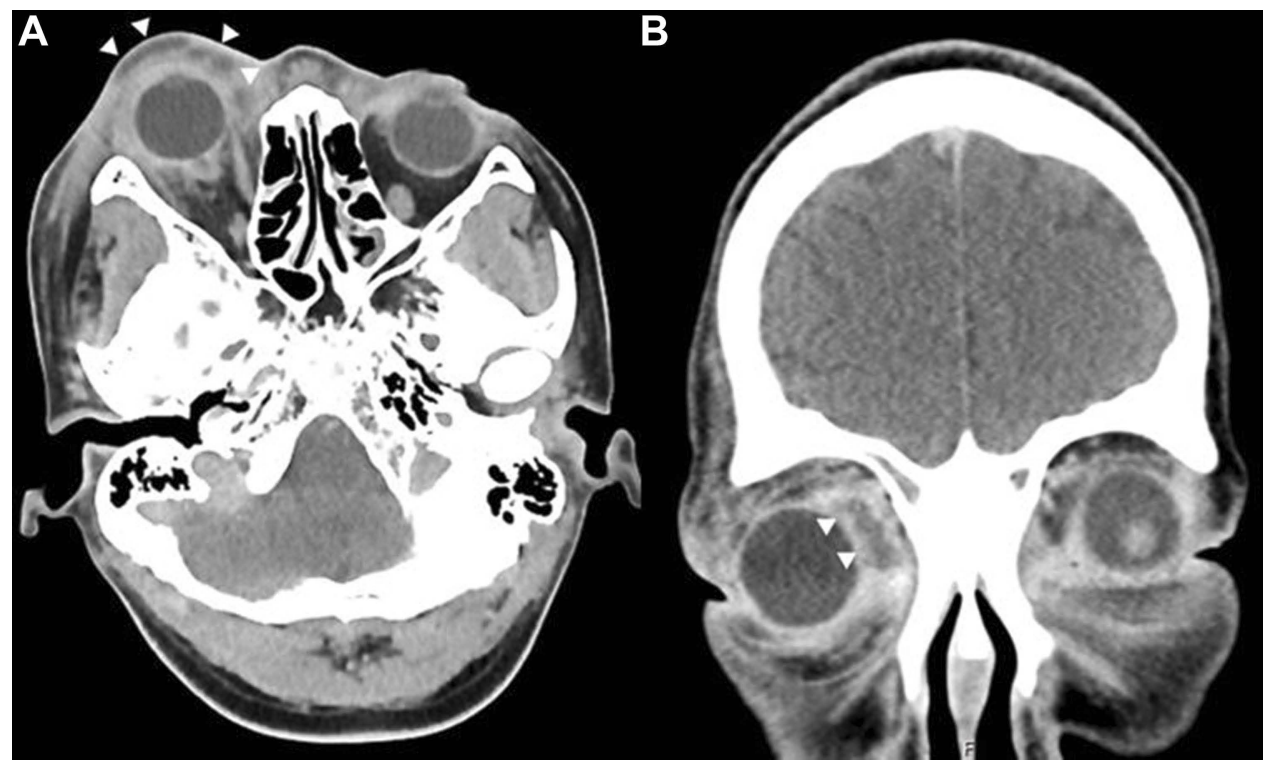

Figure 2 Day 3 (A) axial, (B) coronal CT scan of brain including orbit shows extensive right orbital inflammation with abscess formation, which extended medially from extraconal into intraconal space posterior to eyeball (arrow head).

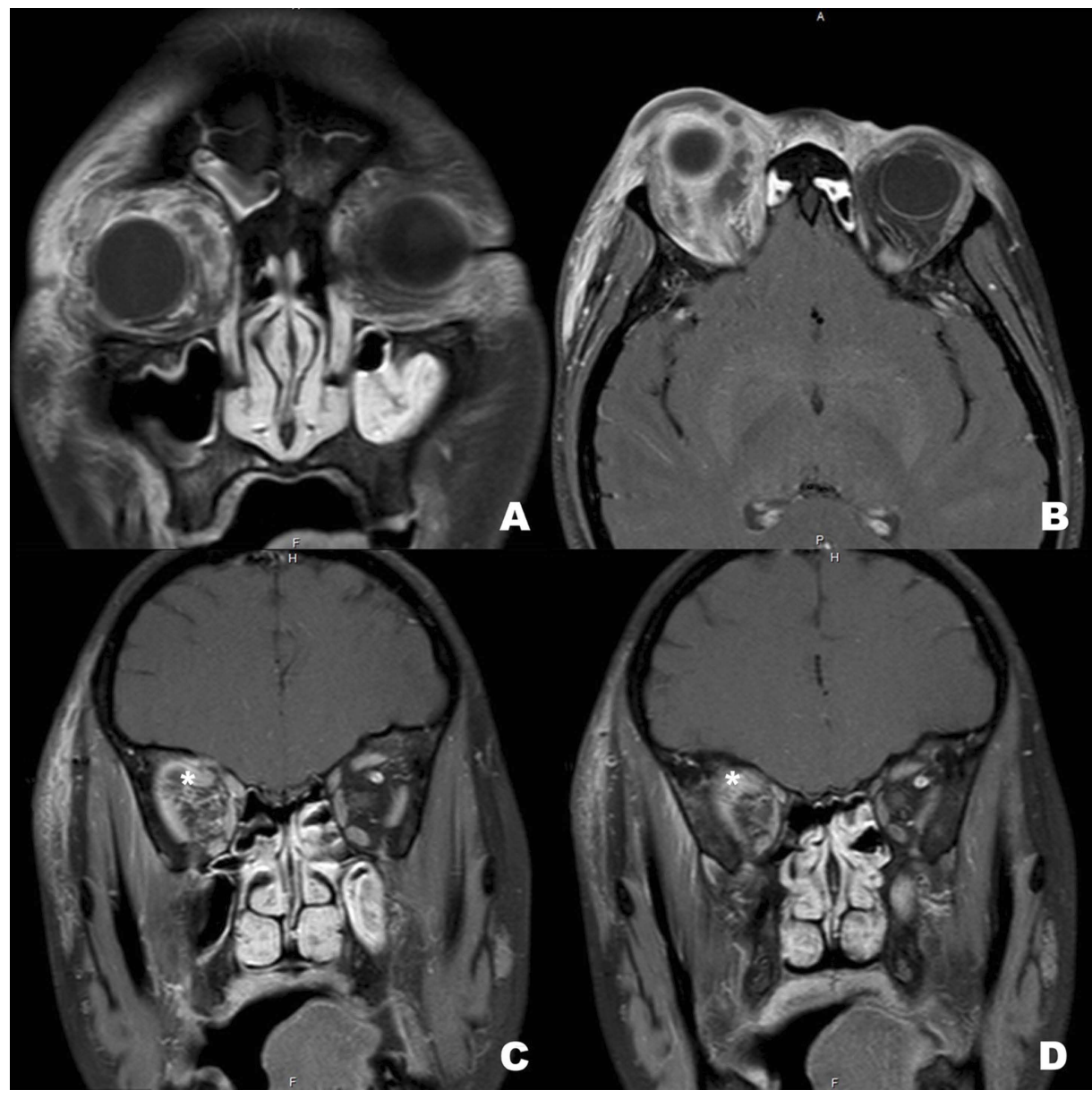

Figure 3 Day 3 (A) coronal and (B) axial TIW with fat suppression MRI scan of brain including orbit showed multiple hyposignal intensity areas with rim-enhancement in preseptal and intraconal spaces. The right superior ophthalmic vein thrombosis is suspicious by intraluminal filling defect with rim-enhancement (star) showing in (C and $\mathbf{D})$ coronal view. 
aureus. The other investigations for organisms were negative.

The patient was treated as for severe orbital cellulitis, and systemic antibiotics were changed to intravenous vancomycin and piperacillin under the supervision of an infectious disease specialist. She underwent wound debridement and orbital abscess drainage twice. IOP was controlled after lateral canthotomy and cantholysis with topical and oral antiglaucoma medications (oral acetazolamide, topical Timolol, and Brimonidine).

The initial workup found hemoglobin of $13 \mathrm{~g} / \mathrm{dL}$, hematocrit $38.6 \%$, white blood cell count 9,900 cell $/ \mathrm{mL}$, platelet $166,000 / \mathrm{mL}$, creatinine $0.84 \mathrm{mg} / \mathrm{dL}$ and blood urea nitrogen $6.6 \mathrm{mg} / \mathrm{dL}$. A peripheral blood smear revealed normochromic microcytic red blood cells and normal morphology of white blood cell and platelets. Urine analyses found no proteinuria, no red blood cells, and white blood cell $0-1$ cell/HPF. Infectious workups were negative for human immunodeficiency virus. Chest x-ray was normal.

The swelling of her eyelid was reduced, visual acuity could be assessed and there appeared to be no light perception. The inflammation on her eye was improved after the second day of drainage. The right eye showed injected conjunctiva, marked chemosis, $3 \times 8-\mathrm{mm}$ epithelial defect at the inferior cornea, but the anterior chamber was unremarkable. IOP was $38 \mathrm{mmHg}$. A relative afferent pupillary defect was noted on her right eye. Fundus examination showed retinal whitening at the posterior pole and a few scattered flame-shaped retinal hemorrhages. The cilioretinal artery presented with retinal whitening along the area of that artery which represented vascular occlusion.

Follow-up MRI brain and orbit with contrast on the fourth days after tertiary care admission was performed to rule out cavernous sinus thrombosis, as physical examination found no improvement of limitation of extraocular movement corresponding to cranial nerve 3, 4 and 6 paresis (Figure 4). Compared to the previous MRI, severe orbital inflammation was improved with no abscess detected but increased degree of right exophthalmos instead. Superior ophthalmic vein thrombosis without cavernous sinus involvement was suspected. Risks and benefits of prescription of either intravenous heparin or oral anticoagulant medication were discussed. It was concluded to have no role in this case. This patient was treated with only antibiotics and monitoring of other complications.

She developed a shaggy-edge macular hole 5 days after initial fundus assessment (Figure 5). Fluorescein angiography revealed cilioretinal artery and retinal artery filling delay with delayed arteriovenous transit time. It also demonstrated that the shaggy-edge window defect angiographic pattern at the macular area consisted of a macular hole (Figures 6 and 7). Optical coherence tomography (OCT) depicted thickening hyper-reflective inner retinal layer (Figure 7D) and macular hole with present of posterior hyaloid membrane (Figure 7E).

This patient was diagnosed with severe orbital cellulitis and abscess formation with combined central retinal vein, central retinal artery and cilioretinal artery occlusion with subsequent macular hole based on retina and imaging findings with increased IOP. Topical antibiotic eye drops, artificial tear drops, topical anti-glaucoma medications and oral acetazolamide were prescribed. The clinical pictures of orbital cellulitis including proptosis, conjunctival injection and chemosis were much improved; also IOP was reduced after the treatment. However, the visual acuity was still no light perception. Hyperbaric oxygen therapy was suggested to increase oxygen supply to retinal tissue but the patient refused.

She responded well to treatment of orbital cellulitis; however, her vision and limited extraocular movement did not fully recover. The intravenous antibiotics were switched to amoxicillin/clavulanic acid before her discharge. Important information from the patient was organized as a timeline (Figure 8).

\section{Ethics approval and informed consent}

The patient's information was collected from reviewed medical records between March 2018 and August 2018 at the Department of Ophthalmology, Phramongkutklao Hospital, Bangkok, Thailand. This case report was approved by the Institutional Review Board, Royal Thai Army Medical Department. This case report involving human data was in accordance with the ethical standards of the institutional and national research committee and with the 1964 Declaration of Helsinki and its later amendments or comparable ethical standards. It was approved by the Institutional Review Board, Royal Thai Army Medical Department. The patient consented to publication of the case (information and pictures) in writing.

\section{Discussion}

Cellulitis after insect bite may initially be confused with an early localized allergic reaction. There is a report ${ }^{5}$ of cellulitis from bee, spider and mosquito, but not black fly. Black flies (Diptera: Simuliidae) ${ }^{6}$ are classified in the same group as flies and mosquitoes. They are distributed widely 


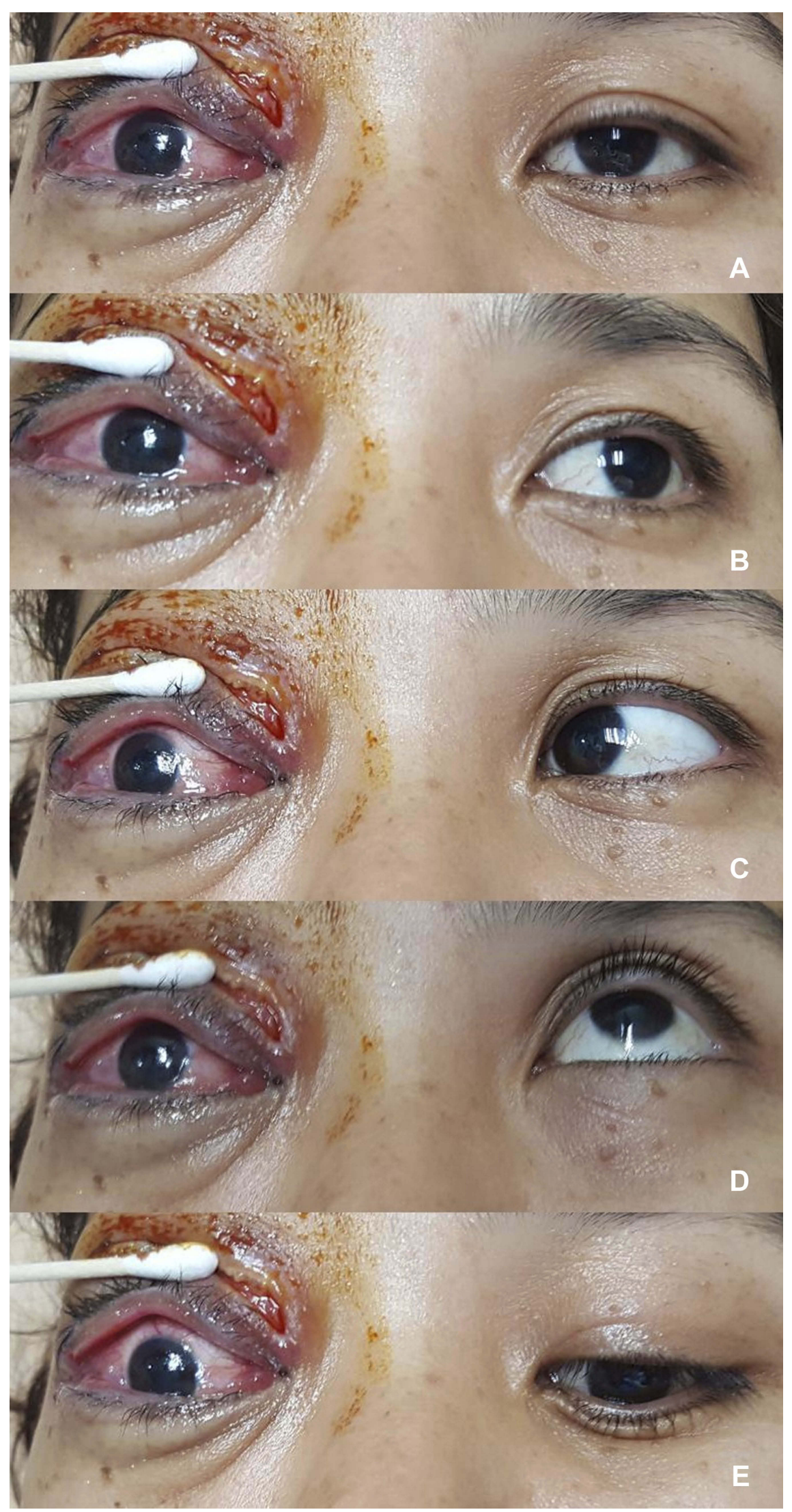

Figure 4 Day 7 limitation of extraocular movement of the right eye in (A) primary position, (B) left gaze, (C) right gaze, (D) up gaze, and (E) down gaze are demonstrated. 


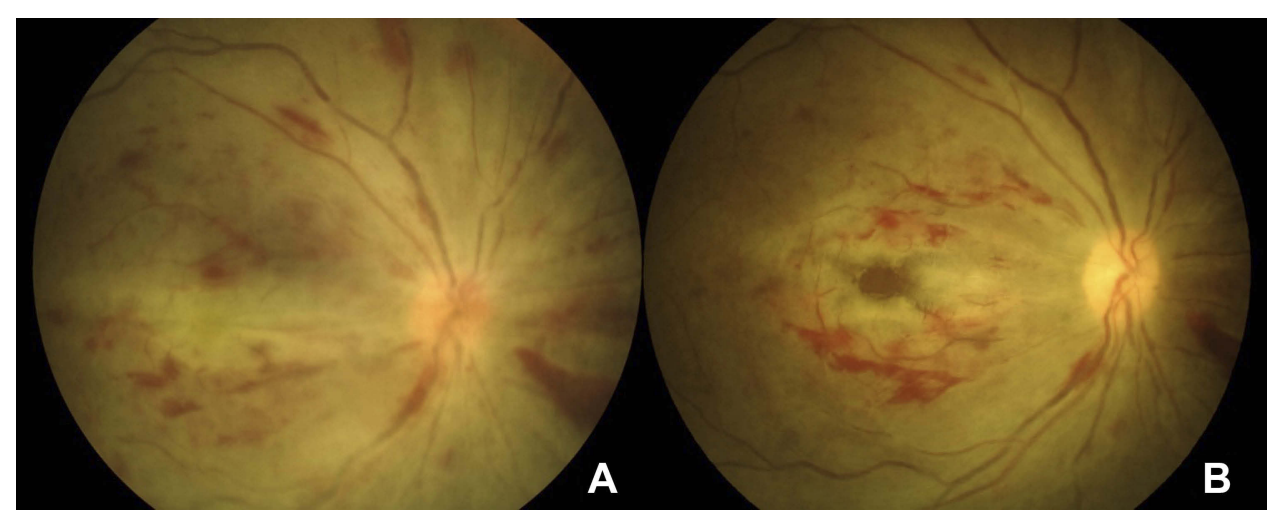

Figure 5 Color fundus photographs show (A) day 5 retinal whitening with scattered flame-shaped retinal hemorrhage, attenuated retinal arteries and venous dilatation representing combined central retinal artery and vein occlusion. There is optic disc swelling. The cilioretinal artery presenting with retinal whitening along the vessel demonstrates cilioretinal artery occlusion. (B) Day 10 a week after treatment by canthotomy and cantholysis to relieve orbital pressure, optic disc swelling, retinal swelling and whitening decreases. However, there is obvious evidence of a macular hole.

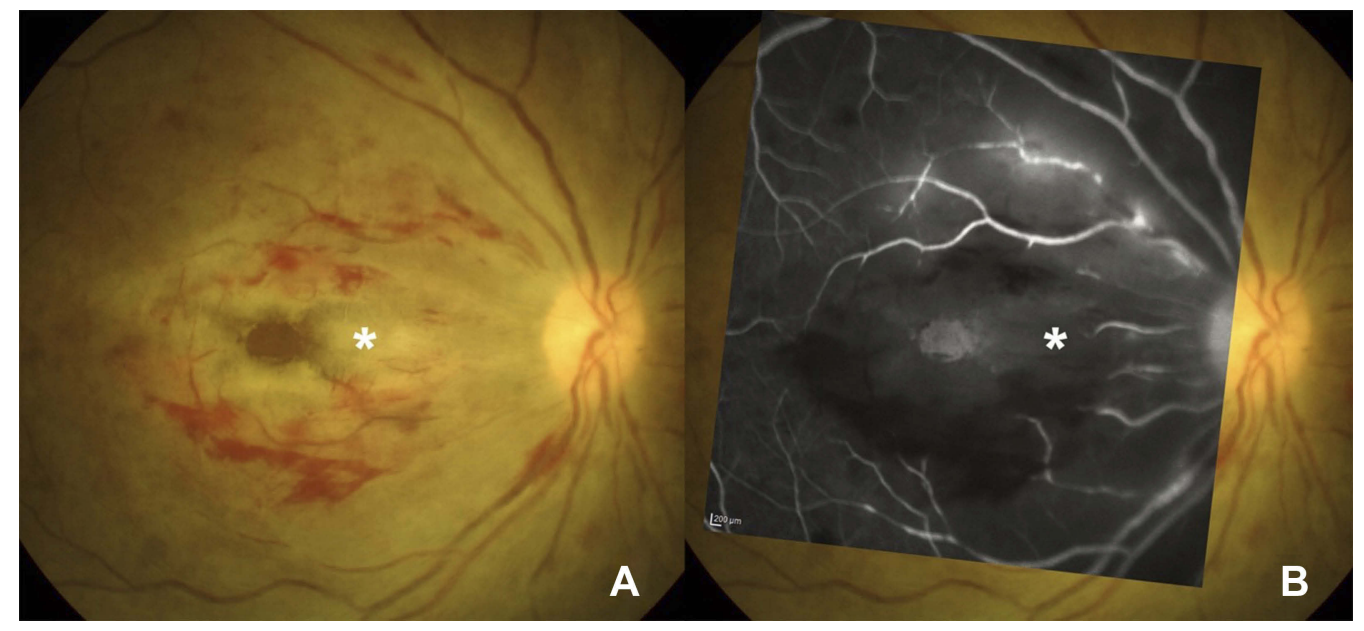

Figure 6 Day 10 fundus photograph shows (A) retinal whitening and hemorrhage in the macular area with macular hole. Cilioretinal artery is occluded (star). (B) Late phase of fluorescein angiography overlying on the fundus photograph reveals vascular filling defect of the arteries around the macula including cilioretinal branch (star).

in Thailand, almost everywhere along the watercourses. The popularity of regional species was highest in the North of Thailand and predominated during winter. They are small, short and a distinct color. Black flies are a threat due to their blood-sucking habits. When the host is bitten, bleeding occurs immediately as well as swelling. People who are allergic to black fly bite would have fever, headache, nausea and asthma which is called "black fever". ${ }^{6}$ In addition, black flies are the carrier of some protozoa and parasites; in particular, adult female black flies are the carrier of Onchocerciasis, which causes blindness in humans from optic nerve involvement. ${ }^{6}$ Furthermore, black fly bites can cause other severe problems in humans since they frequently inflict pain, localized swelling, chronic dermatitis and inflammation accompanied by intense irritation that lasts for several days or even weeks. Additionally, human-biting black fly species are found in large numbers, and cause irritation in domestic environments and to indigenous people.

In this presenting case, a small wound on the right eyelid was found just an hour after being bitten. Although the patient was promptly treated with antibiotics (Cloxacillin), the infection still quickly spread into the orbit within 2 days. This depends on the severity of the pathogen and the immune system of the patient. Several studies ${ }^{1-3}$ state that the most common etiologies of orbital cellulitis involve the infection spreading from neighboring structures such as sinusitis and direct injury of eyelid or orbit which can lead to penetrating infections. The others are hematogenous spreading, opportunistic infections in immunocompromised hosts, dental infection and malignancy. According to our knowledge, there is no known report of orbital cellulitis from black fly bite. 


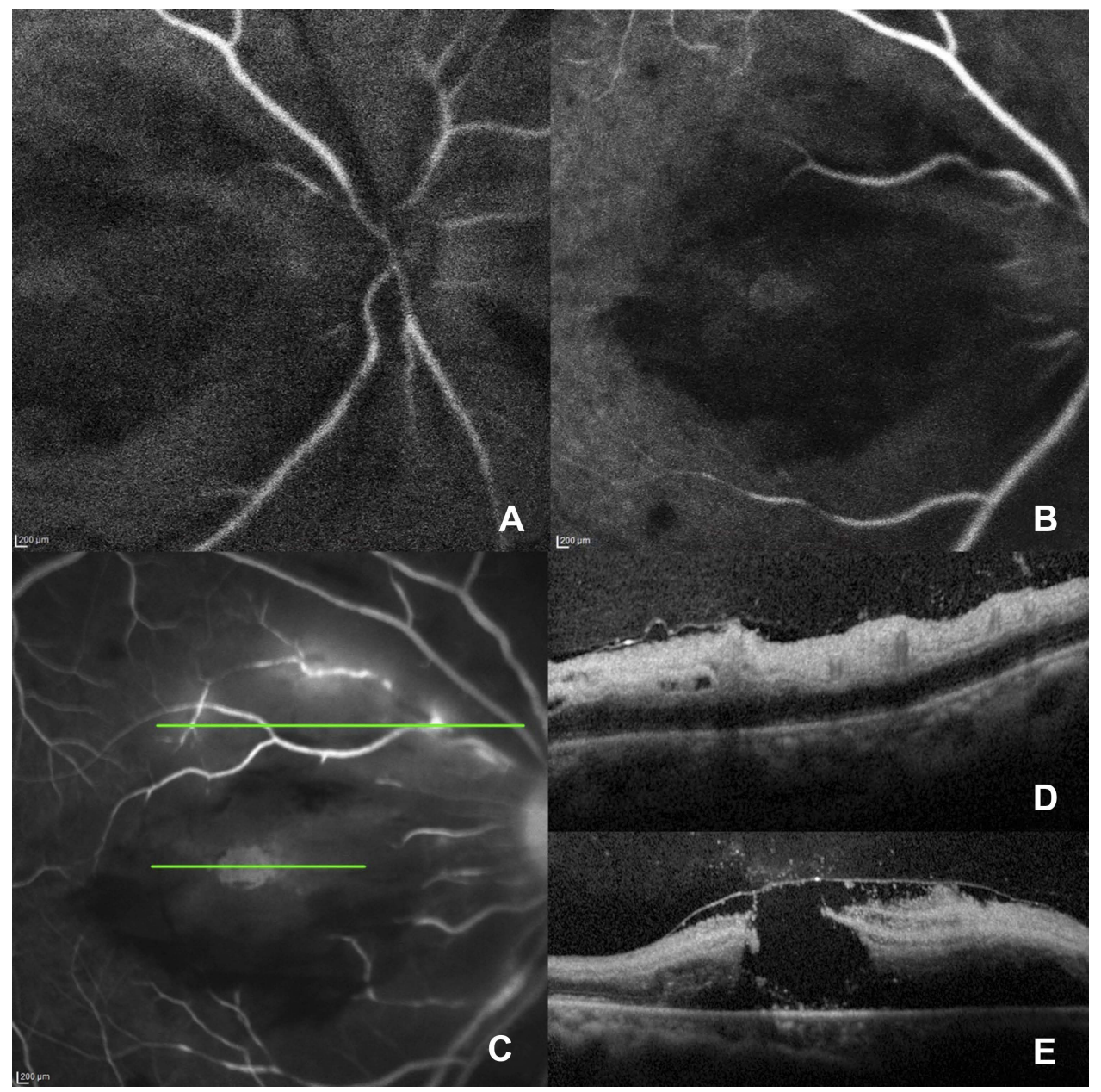

Figure 7 Day 10 fluorescein angiography in the early phase, (A and B) show delayed arterial filling, taken 10 seconds apart. (C) Vascular filling defect and perivascular leakage are detected in late phase with window defect of macular hole. Optical coherence tomography (OCT) corresponding to the upper section on (C) angiography depict (D) the inner retina thickening. This represents inner retinal swelling due to ischemia causing by arterial occlusion. (E) OCT passing the macular on (C) angiography reveals macular hole with the present of posterior hyaloid membrane. Thin strands connecting vitreous and retinal surface are demonstrated, confirming that the macular hole might be from ischemic process rather than traction force.

Thin-slice CT scan with contrast and MRI with fat suppression technique ${ }^{7}$ are very useful imaging methods in the case of orbital cellulitis for identifying the progression of disease, foreign bodies and the presence of subperiosteal abscess or intracranial involvement. Staphylococci and streptococci are the most common organisms in orbital cellulitis ${ }^{1,2}$ as in our case. Loss of vision $^{8}$ occurs in more than $10 \%$ of patients including proptosis resulting in lagophthalmos and corneal exposure, mechanical compression of the optic nerve, severe increased IOP, orbital compartment syndrome, inflammation of the optic nerve and endophthalmitis.

There was a first report about central retinal artery occlusion (CRAO) after orbital inflammation in $2003 .{ }^{8}$ Since then, acute retinal artery occlusion has been a wellknown complication of orbital cellulitis in patients aged between 16 and 60 years, ${ }^{10-14}$ although it is still uncommon. On retinal examination, the appearance of retinal whitening and edema, box-carring and retinal arterial attenuation with redness in the center of macula (cherry red spot) are the hallmark of diagnosis, confirmed by delayed arterial filling in fundus fluorescein angiography. ${ }^{15}$ Treatment of retinal artery obstruction by oral acetazolamide, ocular massage or paracentesis were not successful to restore the vision. Almost all the reported cases had no perception of light. ${ }^{13,14}$ The mechanism of the disease was suddenly increased intraorbital pressure. Therefore, urgent surgical procedures to reduce pressure in the orbit were needed in these situations.

Central retinal vein occlusion (CRVO) $)^{16,17}$ has been reported following inflammatory orbital disease less than CRAO. For the first time in $2007,{ }^{16}$ a child was described 


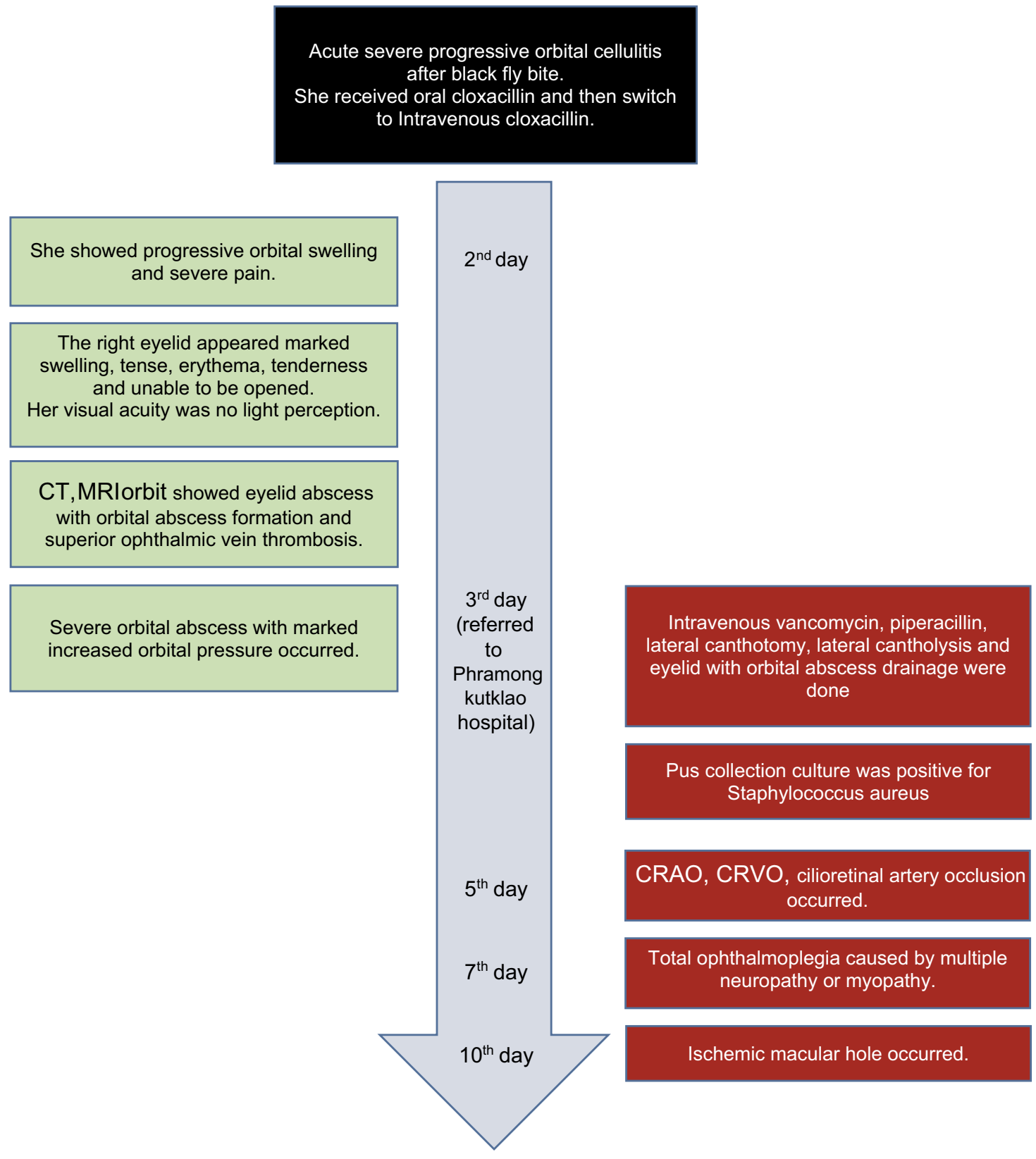

She responded well to treatment of orbital

cellulitis however her vision and limited

extraocular movement did not fully recover.

Figure 8 Timeline of case.

with orbital abscess and increased pressure within the restricted area of the orbit socket. Reducing blood flow in the orbit affected the function of the other structures called the orbital compartment syndrome. CRVO is less severe than CRAO so the vision is slightly better. There was only one report of combined CRAO and CRVO in a pregnant woman, who permanently lost her vision. ${ }^{18}$ In our case, we found combined CRAO, CRVO and also cilioretinal artery occlusion as complications of orbital cellulitis. Unfortunately, even though surgical drainage was successful to decreased intraorbital pressure, the vision was still unrecovered.

Furthermore, there has been a report of ophthalmic artery occlusion after orbital cellulitis ${ }^{19}$ which is more severe than 
retinal artery occlusion. Due to ophthalmic artery supplied anterior segment, retina and choroid, patients with ophthalmic artery occlusion usually have severe permanent visual loss with anterior segment inflammation. There was no sign of anterior segment inflammation in our presenting case. A more complicated case report included ophthalmic artery, cavernous sinus, and superior ophthalmic vein occlusion after fungal infection (mucormycosis) in the orbit, confirmed by MRI. ${ }^{20}$ Similar to our case, the patient developed superior ophthalmic vein thrombosis detected by MRI and was successfully treated by intravenous antibiotics without thrombolysis medication.

Some studies ${ }^{21,22}$ report limited extraocular muscle in orbital cellulitis from cavernous sinus thrombosis. However, cavernous sinus was not shown in our case, and we propose that total ophthalmoplegia could be caused by increased intraorbital pressure or severe inflammation in the orbit causing multiple neuropathy or myopathy.

Our case subsequently developed macular hole after a week of initial fundus examination. Macular hole usually occurs following the traction of the posterior hyaloid. There are a few reports of macular hole following ischemia in the context of inflammation or vascular occlusion. ${ }^{22-24}$ The actual causes of those reports are still controvesial. ${ }^{23-25}$ We believe that our presenting case is a real ischemic macular hole according to the history and fundus finding. Initially the macular was ischemic by combined central retinal artery and cilioretinal artery occlusion with concomitant central retinal vein occlusion. We cannot confirm if there is concurrent choroidal ischemia in this case even though the choroidal thickness seemed thin in the OCT. Markedly ischemic macular might cause inner retinal necrosis followed by the hole. The hole edge appeared shaggy in the color fundus photograph and fluorescein angiogram, which is not typical for vitreomacular traction causing the hole. The OCT also supports our purpose. $\mathrm{T}$ hickening of the inner retina was demonstrated, representing retinal vascular occlusion and the presence of a posterior hyaloid membrane still attached to the foveal and parafoveal area opposing the traction component.

To the best of our knowledge, this case report is the first to identify combined central retinal vein, central retinal artery and cilioretinal artery occlusion followed by ischemic macular hole in a complication of orbital cellulitis. Our case also demonstrated a rare cause of macular hole, supporting by the OCT image. Early detection of increased intraorbital pressure is very important, as it enables earlier appropriate treatments for reducing the risk of progression of multiple retinal vascular occlusion and the involvement of other vital organs such as cavernous sinus which coulddramaticallydeteriorate the prognosis.

\section{Conclusion}

Black flies are a dangerous insect that cause unpredictable infection, especially in the vital organs. Only small bite wound at the eyelid can cause orbital cellulitis and abscess. Rapidly increased orbital pressure from severe orbital cellulitis can cause complications including multiple retinal vascular occlusion. Leaving the condition untreated could lead to loss of vision, therefore close monitoring of complications and prompt treatment would reduce the risk of blindness.

\section{Abbreviations}

CT, computed tomography scan; MRI, magnetic resonance imaging, FFA, fundus fluorescein angiography; OCT, optical coherence tomography; CRAO, central retinal artery occlusion; CRVO, central retinal vein occlusion.

\section{Data sharing statement}

Please contact the corresponding author for data.

\section{Author contributions}

All authors contributed toward data analysis, drafting and revising the paper, gave final approval of the version to be published and agree to be accountable for all aspects of the work.

\section{Disclosure}

Dr Sritatath Vongkulsiri reports personal fees from Novartis and personal fees from Bayer. The authors report no other conflicts of interest in this work.

\section{References}

1. Zhao L, Tang LN, Wu DR, Wang T, Jian TM, Sun FY. [Clinical analysis of orbital and periorbital abscess in 17 patients]. Zhonghua Yan Ke Za Zhi. 2017;53(8):588-593. doi:10.3760/cma.j.issn.04124081.2017.08.006

2. Bajor A, Lang CP, Bültmann E, Framme C, Hufendiek K. [Orbital complication of acute sinusitis: orbital cellulitis in a 10 -year-old child]. Ophthalmologe. 2017;114(4):365-369. doi:10.1007/s00347016-0296-7

3. Hamed-Azzam S, AlHashash I, Briscoe D, Rose GE, Verity DH. Common orbital infections $\sim$ state of the art $\sim$ part I. J Ophthalmic Vis Res. 2018;13(2):175-182. doi:10.4103/jovr.jovr_199_17

4. Gagnier JJ, Kienle G, Altman DG, et al. The CARE guidelines: consensus-based clinical case reporting guideline development. Glob Adv Health Med. 2013;2(5):38-43. doi:10.7453/gahmj.2013.008

5. Derlet RW, Richards JR. Cellulitis from insect bites: a case series. Cal J Emerg Med. 2003;4(2):27-30. 
6. Srisuka W, Takaoka H, Otsuka Y, et al. Biodiversity, seasonal abundance, and distribution of blackflies (Diptera: Simuliidae) in six different regions of Thailand. Parasit Vectors. 2017;10(1):574. doi:10.1186/s13071-017-2494-9

7. Weber AL, Mikulis DK. Inflammatory disorders of the paraorbital sinuses and their complications. Radiol Clin North Am. 1987;25:615-630.

8. Hartstein ME, Steinvurzel MD, Cohen CP. Intracranial abscess as a complication of subperiosteal abscess of the orbit. Ophthal Plast Reconstr Surg. 2001;17:395-397.

9. Bhola RM, Dhingra S, McCormick AG, Chan TK. Central retinal artery occlusion following staphylococcal orbital cellulitis. Eye (Lond). 2003;17(1):109-111. doi:10.1038/sj.eye.6700250

10. Okamoto Y, Hiraoka T, Okamoto F, Oshika T. A case of subperiosteal abscess of the orbit with central retinal artery occlusion. Eur J Ophthalmol. 2009;19(2):288-291.

11. Coşkun M, Ilhan Ö, Keskin U, et al. Central retinal artery occlusion secondary to orbital cellulitis and abscess following dacryocystitis. Eur J Ophthalmol. 2011;21(5):649-652. doi:10.5301/EJO.2011. 6493

12. Proctor CM, Magrath GN, de Castro LE, Johnson JH, Teed RG. Orbital cellulitis complicated by central retinal artery occlusion. Ophthal Plast Reconstr Surg. 2013;29(2):e59-e61. doi:10.1097/ IOP.0b013e31826b7760

13. Ismi O, Vayisoglu Y, Dinc E, Yildirim O, Unal M. Central retinal artery occlusion and irreversible blindness due to paranasal sinus infection in a pregnant woman. J Craniofac Surg. 2014;25(6):e557e559. doi:10.1097/SCS.0000000000001083

14. Park CH, Jee DH, La TY. A case of odontogenic orbital cellulitis causing blindness by severe tension orbit. J Korean Med Sci. 2013;28 (2):340-343. doi:10.3346/jkms.2013.28.2.340

15. American Academy of Ophthalmology. Basic and Clinical Science Course. Section 12: Retina and Vitreous. Colin A McCannel: AAO; 2018-2019.
16. Das JK, Choudhury BD, Medhi J. Orbital abscess and hemi-occlusion of the central retinal vein in a child. Orbit. 2007;26(4):295-297. doi:10.1080/01676830601169155

17. Vohra V, Chawla H, Gupta M. Venous occlusion in a case of orbital cellulitis. Korean J Ophthalmol. 2016;30(6):483-484. doi:10.3341/ kjo.2016.30.6.483

18. Komolafe OO, Ashaye AO. Combined central retinal artery and vein occlusion complicating orbital cellulitis. Niger J Clin Pract. 2008;11 (1):74-76

19. Alvi NP, Mafee M, Edward DP. Ophthalmic artery occlusion following orbital inflammation: a clinical and histopathological study. Can J Ophthalmol. 1998;33(3):174-179.

20. Hatipoglu HG, Gurbuz MO, Yuksel E. Restricted diffusion in the optic nerve and retina demonstrated by MRI in rhino-orbital mucormycosis. J Neuroophthalmol. 2009;29(1):13-15. doi:10.1097/ WNO.0b013e318183bde4

21. Bouraoui R, Bouladi M, Ben Romdhane B, Limaiem R, Mghaieth F, El Matri L. Ophthalmic artery occlusion with total ophtalmoplegia and anisocoria revealing cavernous sinus thrombosis. Tunis Med. 2016;94(2):145-147.

22. Allegrini D, Reposi S, Nocerino E, Pece A. Odontogenic orbital cellulitis associated with cavernous sinus thrombosis and pulmonary embolism: a case report. J Med Case Rep. 2017;11(1):164. doi:10.1186/s13256-017-1309-0

23. Karim-Zade K, Bilgic A, Bartz-Schmidt KU, Gelisken F. Horseshoelike macular tear following recurrent branch retinal vein occlusion. Graefes Arch Clin Exp Ophthalmol. 2007;245(8):1221-1223. doi:10.1007/s00417-006-0500-6

24. Panos GD, Papageorgiou E, Kozeis N, Gatzioufas Z. Macular hole formation after toxoplasmic retinochoroiditis. BMJ Case Rep. 2013;2013:bcr2013008915. doi:10.1136/bcr-2013-008915

25. Panos GD, Gatzioufas Z. Macular hole formation after toxoplasmic retinochoroiditis: coincidence or rare complication? Int Ophthalmol. 2013;33(3):219. doi:10.1007/s10792-013-9770-2

\section{Publish your work in this journal}

The International Medical Case Reports Journal is an international, peer-reviewed open-access journal publishing original case reports from all medical specialties. Previously unpublished medical posters are also accepted relating to any area of clinical or preclinical science. Submissions should not normally exceed 2,000 words or 4 published pages including figures, diagrams and references. The manuscript management system is completely online and includes a very quick and fair peer-review system, which is all easy to use. Visit http://www.dovepress.com/testimonials.php to read real quotes from published authors. 